\title{
Dual Energy CT - A diagnostic boon
}

\author{
AL Mohammad MA*, Hao H, Bo L and Bin SH \\ Renmin Hospital, the First Affiliated Hospital, Nanjing Medical University, Jiangsu Province, China
}

\begin{abstract}
Dual Energy CT (DECT) has been evidenced to be a boon within the field of diagnostic imaging recently. It functions by utilizing a spectrum of tow photons, thus it's referred to as 'dual energy' CT. Visualizations that are intricate in structural and purposeful detail are possible by DECT due to its capability of material differentiation and improved detection of iodine at low energies. Due to its ability to differentiate different materials, the requirement for recurrent examinations and exposure to radiation for the patient is reduced, thereby creating it a safer choice for diagnosis. more during this article, the applications of DECT in clinical observe for the characterization of excretory organ calculi, liver lesions, thoracic imaging, oncology imaging and artifact reduction. has been represented. Each coin has two sides, thus, DECT has its own limitations too, that is mentioned more in the article.
\end{abstract}

\section{Introduction}

Computed tomography scan (CT-scan) is that servant for contemporary for radiology imaging, that accounts for roughly sixty-five million adult scans within u. s. each year [1]. Advances in technology like quicker scan times, thin slices, multiple reformat, and 3D reconstruction have helped in revolutionizing the scope of CT. Dual-energy CT(DECT), has been one among the foremost promising developments in radiology in recent history [2]. It yields increased image contrast resolution by at the same time exploit scan information at two different energy levels of x-ray tube - usually, eighty $\mathrm{kV}$ and one hundred forty $\mathrm{kV}$. it's referred to as 'dual-energy' because it utilizes spectra of tow photons; so, DECT is additionally mentioned as "spectral CT." In clinical observe, by changing the voltages of one $\mathrm{x}$-ray tube or by running tow tubes at totally different voltages, tow totally different spectra are unit generated, and spectral data is obtained from tow absorption measurements with normal CT detectors $[3,4]$. Dual-energy CT techniques want to distinguish substances like iodine, calcium, and acid crystals from soft tissues. This capability for material differentiation and improved detection of iodine at low energies, has created DECT imaging and post process to relinquish complicated visualizations that are tangled in structural and purposeful detail, that permit isolation and quantification of tissues and disease processes in higher detail compared to traditional singleenergy CT imaging - frequently while not magnified radiation doses to patients $[2,4,5]$. Due to this property DECT completely differentiate between different materials over other medical modalities, it would like for multiple examinations and recurrent exposure to radiation is reduced, thereby, being a bonus by reducing the accumulative dose for the patient $[4,6,7]$ to permit a differentiation on DECT, the tissue or contrast medium within the examined area should have spectral properties - that is, variations in $\mathrm{x}$-ray attenuation at totally different gauge photon energies. The $\mathrm{x}$-ray attenuation is caused by the Compton effect, coherent scatter, and also photoelectrical effect. of those properties, the photoelectrical effect powerfully caused of the atomic number of the material. as a result of the photoelectrical effect powerfully will increase with atomic number, most of the atoms within the physical structure (i.e., hydrogen, carbon, nitrogen, and oxygen) have a rather weak photoelectrical effect. Some ions within the body like calcium or magnesium have a somewhat stronger result, whereas the photoelectrical effect of contrast medium (i.e., iodine, barium, or xenon) is extremely sturdy $[4,8]$ let's say, DECT images are often wont to differentiate kidney stones that contain calcium from kidney stones that contain uric acid or to differentiate brain hemorrhages related to tumors from those with noncancerous causes $[9,10]$. This new technique permits careful characterization of non-tissue materials and reduces imaging artifacts caused by metal devices [11].

DECT was 1st discovered within the Nineteen Seventies. it absolutely was solely when this that DECT scanners became wide offered for clinical use $[12,13]$. DECT has customary workstations permitting clinicians to deal with common queries in each acute (emergency) and chronic disease settings [14,15] let's say, automatic digital subtraction of bone from DECT angiography images in DECT digital computer software system when the process is finished, even in complicated anatomic regions [14].

Dual-energy techniques additionally could also be wont to distinguish substances uric acid crystals in the kidney. Characterization of renal calculi helps to work out whether or not a patient ought to be treated with medical management, lithotripsy, or open ablation $[6,16]$. rising applications not nonetheless in widespread clinical use embrace neuroradiology and oncology imaging for tumor detection, characterization, and watching throughout and when medical care $[4,12,13,15]$.

\section{DECT in practice}

\section{Excretory organ stone formation}

Additionally, to symptoms, size, and placement, the component of excretory organ stones its essential for acceptable clinical management.

Correspondence to: ALMohammad MA, Renmin Hospital, the First Affiliated Hospital, Nanjing Medical University, Jiangsu, China, E-mail: manaralmohammed@yahoo.com

Key words: DECT, advanced, radiodiagnosis

Received: March 02, 2018; Accepted: March 19, 2018; Published: March 23 2018 
it's necessary for the medical specialist to differentiate uric acid stones (treated medically) from non-uric acid stones (treated with invasive strategies like extracorporeal shockwave lithotripsy or transdermal nephrolithotripsy). though uric acid stones tend to own lower attenuation values than non-uric acid stones (i.e., struvite, cystine, and calcium) on DECT, they will be troublesome to differentiate the cause of overlap in attenuation values [5].

Conventional single-energy CT doesn't reveal the chemical composition of excretory organ stones, however, DECT will do therefore with post-process software system during which the quantitative relation of attenuation between the high- and low energy information sets are often calculated $[3,5,6,17]$.

DECT helps in differentiating excretory organ calculi by analysis of underlying composition, as uric acid stones are composed of lighter components (hydrogen, carbon, oxygen, and nitrogen) whereas non-uric acid stones are composed of heavier components (calcium, phosphorus, and sulfur) $[5,18,19]$ (Figure 1).

Conversely, non-uric acid stones demonstrate exaggerated attenuation at eighty $\mathrm{kVp}$ because Uric acids stones demonstrate exaggerated photons attenuation at a hundred and forty $\mathrm{kVp}$ as a result of the attenuation is driven primarily by Compton scatter (due to lighter $30 \%$ chemical composition). Conversely, non-uric acid stones demonstrate exaggerated attenuation at eighty $\mathrm{kVp}$ because of exaggerated electricity impact contribution from the heavier parts nearing the $\mathrm{K}$-edge of the metallic element $(\mathrm{Z}=20)$. victimization the two-material (basis pair) decomposition approach on DECT, water and iodine image datasets square measure generated. Stone look on water image solely suggests acid calculi, whereas look on every water and iodine image indicate non-uric acid stones (Figure 2). Effective Z (Zeff) pictures square measure AN alternate approach for characterizing internal organ calculi and should be achieved in the offline computing device. Zeff weighs each the attenuation and number of bound materials, thereby serving to in characteristic the materials that are predominant among mixed stones.
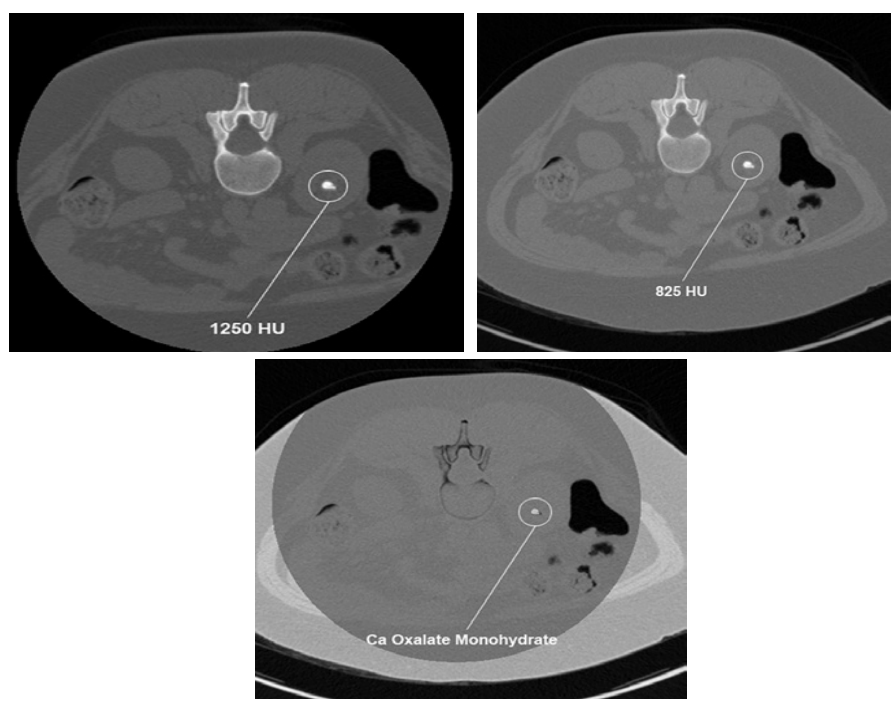

Figure 1. DECT characterization of excretory organ stones. A. DECT axial image shows left excretory organ calculus (stone) with attenuation of $825 \mathrm{HU}$ at one hundred forty $\mathrm{kVp}$. B. This image has $1250 \mathrm{HU}$ attenuation at eighty $\mathrm{kVp}$. C. The stone was firm to be composed of calcium oxalate monohydrate victimization DECT post process algorithms. Reprinted with permission from Coursey CA, Nelson RC, Boll DT, et al. Dual-energy multidetector CT. Radiographic. 2010;30(4): 1045.Uric acids stones demonstrate exaggerated photons attenuation at a hundred and forty $\mathrm{kVp}$ as a result of the attenuation is driven primarily by Compton scatter (due to lighter $30 \%$ chemical composition).
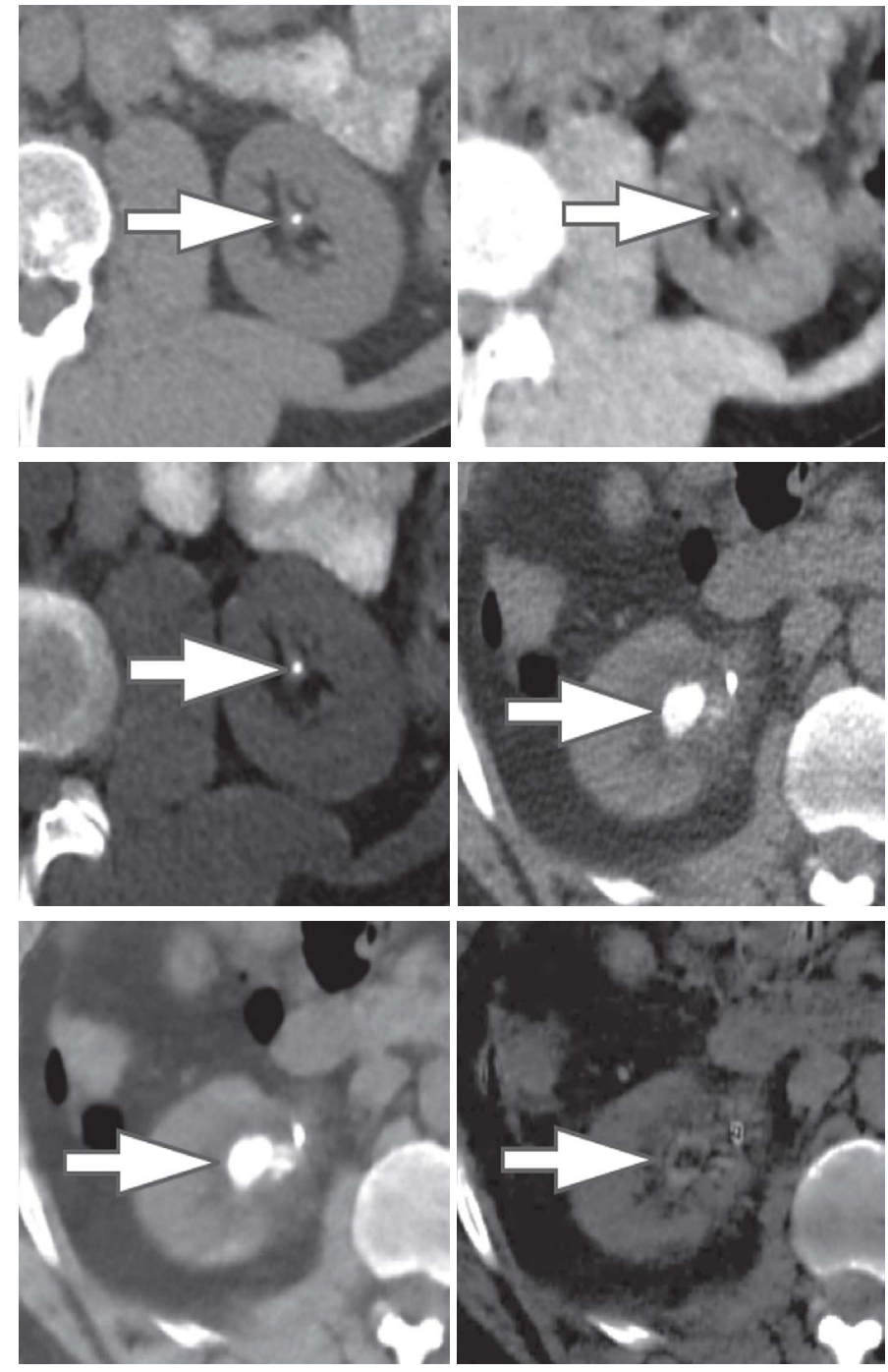

Figure 2. 45-year-old man (top row) and 60-year-old man (bottom row) with renal lithiasis typical true unenhanced image (A), water material decomposition image (B), and iodine material decomposition image (C) demonstrate a non-uric acid stone, that's hyperdense on every water and iodine pictures (arrows) in single offer DECT (GE). at intervals, the second patient, typical true unenhanced image (D), water image (E), and iodine image (F) demonstrate a mixed stone that is predominantly acid in composition (arrows). The stone is not hyperdense on iodine pictures aside from its peripheral rim (F). Our experience has shown that acid stones typically demonstrate a rim of Ca deposition.

Low Zeff is commonly seen in acid stones, whereas high Zeff is seen in non-uric acid stones. The algorithm for viscus stones in DECT assumes that everyone voxel is a mixture of a metallic element, uric acid, and water. Calculi with X-ray attenuation profiles like metallic element appear blue whereas stones with profiles like acid appeared (Figure 3).

Iodine contrast-subtracted virtual unenhanced DECT images can also facilitate to differentiate between kinds of excretory organ pathologies, like hemorrhagic cysts and solid tumors [5]. hemorrhagic excretory organ lesions are visible on each contrast-enhanced and virtual unenhanced DECT images. element contrast-enhanced images permit color-coded quantification of iodine among a lesion [10].

\section{Liver lesion characterization}

Evaluation of little liver lesions on typical CT is typically a diagnostic plight. Sub centimeter lesions area unit typically very little 


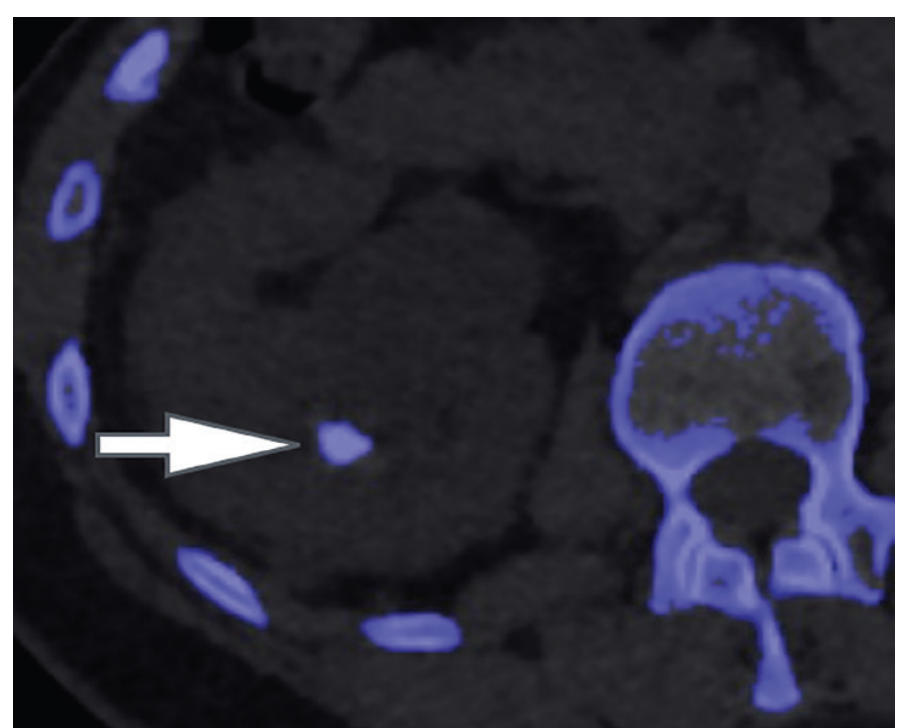

Figure 3. A 57-year-old woman with a metal urinary organ stone. With twin supply DECT (Siemens), metal stones appear blue (arrow) whereas acid stones appear red.

to characterize, leading to the necessity of a lot of investigations with tomography or diagnostic test. Cysts are typically differentiated from metastases (small hypodense masses) on iodine pictures in Associate in the extremely DECT scan as a result of a metastasis will uptake iodine whereas a cyst will not (Figure 4) [20].

Evidence for DECT iron quantification of viscus tissue is prescribed. whereas specificity is sort of good, sensitivity is extremely poor that it's been counseled to avoid its use for this application, particularly at low levels of iron [20].

Detection and characterization of improvement in lesions by DECT in all probability reduce the need for traditional short interval follow-up studies, decrease the need for supernumerary biopsies, and improves screening for hypervascular tumors in cirrhotic patients [20].

\section{Oncology imaging}

DECT has several applications in every initial and post-treatment medicine imaging. By increasing lesion eminence, growth margins square measure higher printed, thus, property accuracy in measurement of the scale of lesions (Figure 5) [5].

Accuracy in a measure is extremely necessary for guiding management, notably for patients in clinical trials throughout that growth response is looking forward to serial measurements per the various criteria for analysis of tumors [21,22]. due to the potential ability of quantitative iodine maps for the analysis and quantification of the viability of neoplasm, DECT has an extra potential for assessment of the response to treatment twenty-one [22]. this is often a rousing development that permits for characterization of neoplasm at a purposeful level that anticipates any modification in size [21], though this technique wants validation and standardization. to boot to increasing lesion eminence, low Kev and material density pictures can also offer correct image of regional extent of malady and relationships to adjacent vasculature that's helpful to guide treatment prepare [22]. curiously, iodine maps can facilitate in differentiating a neoplasm from bland coagulum through qualitative and quantitative demonstration of iodine among the clot. This differentiation is diagnostic in staging hepatocellular and internal organ malignant neoplastic disease [22]. DECT can facilitate in assessing the response to treatment involving therapies targeted to express regions, such as ablation (e.g., radiofrequency, microwave, cryo), targeted irradiation (e.g., protons), and intra-arterial medical aid (e.g., selective internal irradiation [SIRT] and transarterial chemoembolization [TACE]) $[23,21,22]$. Iodine maps obtained sort of a shot once radiofrequency ablation has shown higher lesion conspicuity and internal homogeneity of the ablation zone, providing an additional profit for assessing the security once radiofrequency ablation. In our observations, we've observed that iodine pictures are helpful not only sort of a shot once the procedure but to boot for following from patients undergoing internal organ microwave/radiofrequency ablation and internal organ cryo/ radiofrequency ablation (Figure 6).

\section{Thoracic imaging}

Thoracic DECT examinations optimize medium injection parameters, in conjunction with the utilization of a saline chaser bolus, can cut back artifacts, improve quality of an image, and increase diagnostic accuracy. High-concentration (i.e., > three hundred mg I/ $\mathrm{mL}$ ) iodine-based medium is typically suggested for DECT studies to
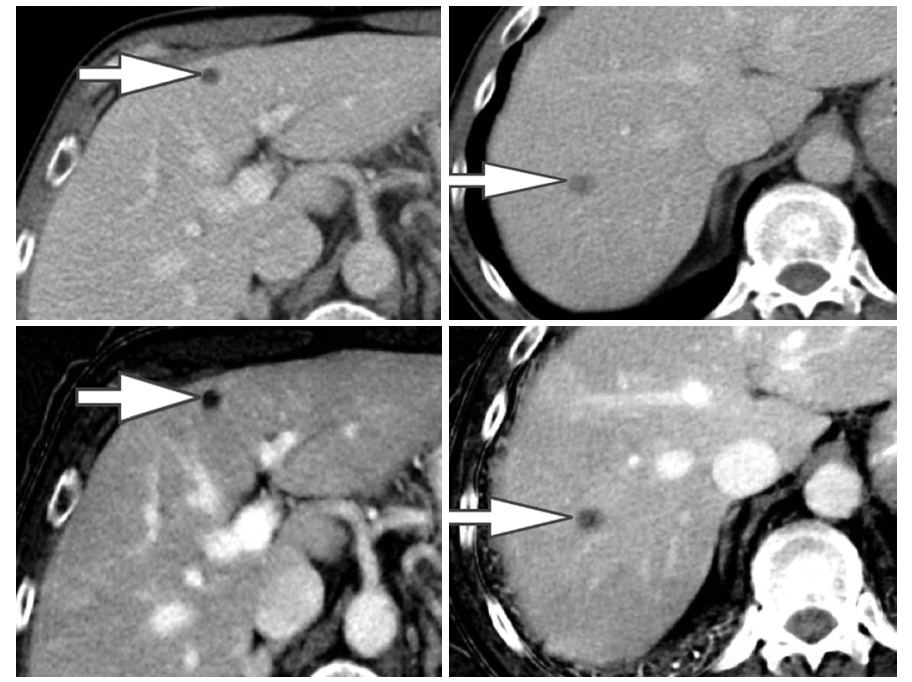

Figure 4. A 65-year-old man with a history of the malignant neoplastic disease. Contrastenhanced Porto venous section single energy $\mathrm{CT}$ pictures show tow small rounded hypodense lesions in internal organ segments IV (arrow in A) and VII (arrow in B), that are really similar in look. Iodine material decomposition pictures reveal the varied nature of the tow lesions. the phase IV clinical trials lesion demonstrates no uptake of iodine and is so a cyst (arrow in C), whereas the section VII lesion demonstrates iodine uptake (arrow in D) and is so a metastasis.
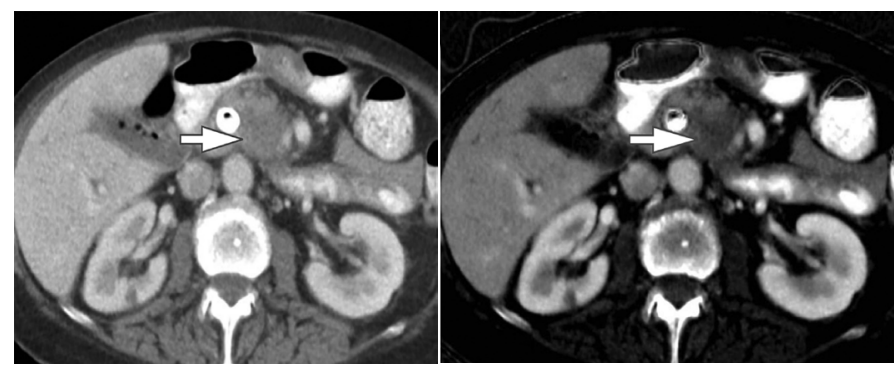

Figure 5. A. 72-year-old man with a history of jaundice treated with biliary tube placement (A) Single-energy CT pictures, that place confidence in variations in Hounsfield units, show degree ill-defined area of heterogeneous improvement among the uncinate methodology of the secretor (arrow). (B) Iodine material decomposition pictures (GE), that demonstrate the actual uptake or concentration of iodine in tissues, facilitate to higher characterize and describe the extent of this hypo enhancing mass (arrow), compatible with ductal carcinoma. to boot note but the whole reduction in twin energy CT results in a higher image of the metal tube containing refluxed oral contrast media. 


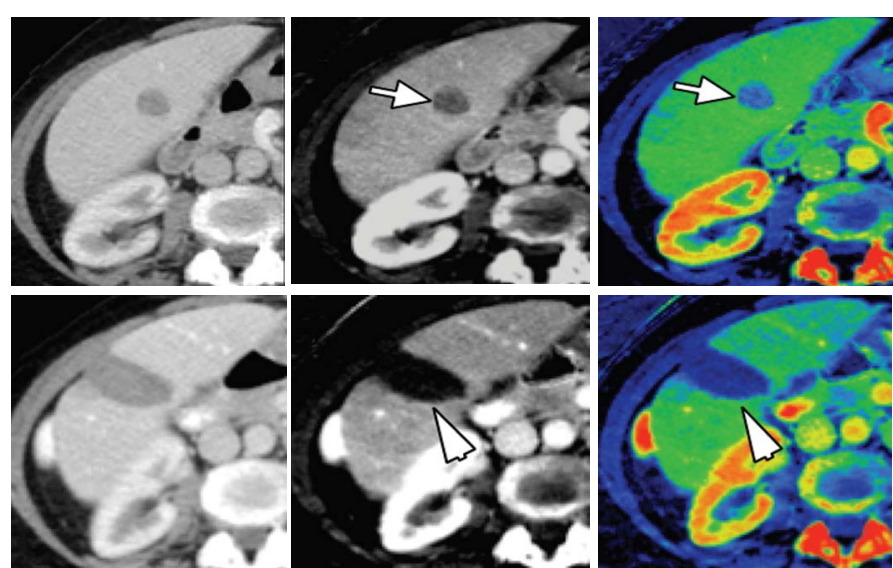

Figure 6. A 62-year-old girl with a history of a malignant neoplastic disease and a solitary liver metastasis. (A) Single energy CT image learned at a hundred and forty kVp, (B) material decomposition iodine image, and (C) color overlay iodine image demonstrate the solitary heterogeneous liver lesion in part V. Material decomposition iodine pictures $(\mathrm{B}, \mathrm{C})$ demonstrate moderate uptake of iodine (arrows), suspicious for metastasis, that was later confirmed at assay. Following radiofrequency ablation, the $140 \mathrm{kVp}$ pictures(D) still show heterogeneous attenuation among the metastasis, raising suspicion for incomplete ablation or repetition. However, iodine pictures (E, F) show no uptake of iodine (arrowheads), excluding recurrence/ incomplete ablation; the heterogeneous look of the lesion is maybe going due to post-treatment trauma.

boost the differentiation of iodine by the dual-energy algorithms once method [22].

There are many pectoral imaging protocols which are able to profit DECT. let's say, iodine improvement maps offer physiological information regarding the introduction of iodine at intervals the internal organ parenchyma, which may be helpful in identification introduction deficits because of respiratory organ emboli or various conditions like emphysema [24]. Initial studies counsel that quantification of the degree of iodine improvement might even be helpful in differentiating between benign and malignant internal organ nodules [15]. DECT has to boot been useful for patients are unable to spice up their arms on top of heads as a result of post method of DECT pictures can limit artifacts because of beam hardening caused by the arms. Similarly, streak artifacts because of metal prostheses in, let's say, the shoulder or the spine, are usually reduced with DECT. Moreover, as a result of iodine improvement is larger at lower Kev, a lower dose of iodine medium is usually used once imaging with DECT (Figure 7) if medically indicated $[15,25,26]$.

\section{Pulmonary embolism}

CT angiography of the pneumonic arteries (CTPA) could also be a sensitive and speedy methodology for detecting emboli at intervals the pneumonic arteries. it's replaced typical scintigraphy methods for ventilation/ perfusion imaging in AN passing majority of patients. optimum single-energy CTPA desires shut attention to the temporal order of image acquisition once the excellence has been administered. Inappropriate temporal order of acquisition of the image may result in a non-diagnostic examination, that's means less common with DECT than with single energy CTPA. to boot, distinction improvement in smaller branches of the pneumonic arteries is sometimes higher on DECT than on single-energy CTPA. Post method of DECT pneumonic roentgenography can generate virtual non-contrast pictures, customary CPTA pictures, and iodine distribution map that will show if their square measure any perfusion deficits. Deficits caused by embolism square measure typically wedge-shaped. Thus, DECT has the potential to increase the accuracy of identification for pneumonic emboli. to boot, the size of the perfusion deficit is prognostic as larger perfusion deficits square measure involving poorer prognosis $[15,26,27]$.

\section{Pulmonary nodules}

Initial studies have according to that single part DECT that a hundred seconds once administration of contrast material may facilitate to differentiate between benign and malignant nodules 24 . the chosen region of interest in DECT is identical in each of the postprocessed pictures but won't be so for single energy dynamic CT. Iodine improvement at eighty kilovolts is regarding double that at one hundred forty kilovolts, that's usually used for single energy CT. Iodine distribution maps provide a quantitative assessment of iodine uptake. For of those reasons, DECT has the potential to outdo single energy CT for diagnostic accuracy for malignancy in internal organ nodules and at an identical time reducing radiation dose [15].

\section{Routine chest imaging}

Chest CT examinations are performed for form of reasons, alongside staging of cancer, assessing response to treatment, characterization of abnormalities, and for symptomatic patients with traditional photography pictures [26]. in addition to providing customary CT pictures obtained from routine chest single-energy CT, a contrast media increased routine DECT permits synchronic assessment of the pneumonic arteries and provides just about an identical information as that obtained through an embolism protocol [15]. Iodine improvement maps offer physiological information and build it easier to acknowledge pathologies like pneumonic pathology (Figure 8), respiratory illness and pathology [26]. to boot, characteristic patterns of introduction deficits are concerning different internal organ pathologies, like pulmonary emphysema and air trappings. DECT has similar blessings for cancer staging, response to medical aid and malady looking. the property, so iodine uptake, can diminish in neoplasms that area unit responding to

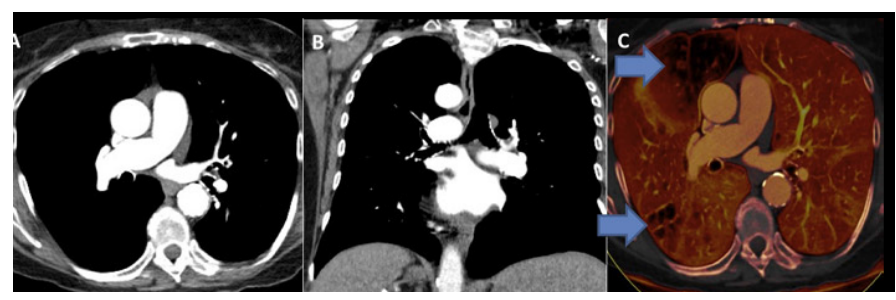

Figure 7. Patient with one urinary organ and shortness of breath had scattered respiratory disease and pathology on chest pictures. The patient underwent a DECT embolism protocol CT with solely twenty-five ccs of contrast material (generally 80-100 cc of contrast material is used). customary CT pictures at sixty Kev (a and b) improvement distinction improvement in pneumonic arteries (main, lobar, segmental, and subsegmental) pneumonic blood volume image (C) shows scattered defects (arrows) in line with areas of pulmonary emphysema (these defects would have reduced the sensitivity and specificity of nuclear V:Q scan).

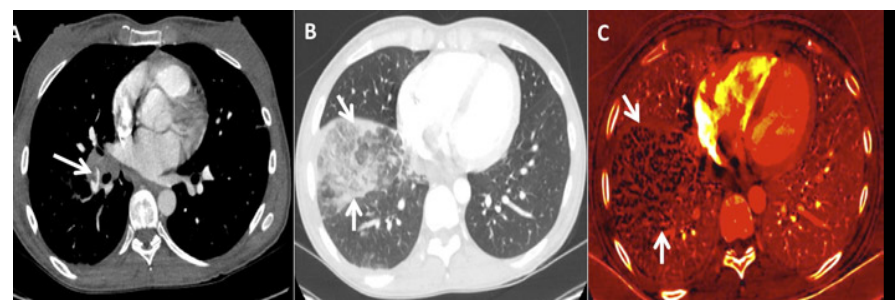

Figure 8. customary CT pictures (A and B) at sixty Kev demonstrate occlusive filling defect (arrows) among the right interlobar artery pulmonarias and consolidation at intervals the correct lower lobe. pulmonary blood volume image (C) demonstrates a neighborhood of reduced iodine uptake (arrows) that's larger than the size of consolidation. This opacity is in line with pulmonary infarction. 
medical aid before any shrinkage of tumor happens. in addition, DECT can differentiate between iodine improvement and calcification, which might be observed in liquid body substance nodes in non-contrast pictures and maybe a cause confusion in single-energy CT [23].

\section{Vascular imaging}

CT angiography (CTA) involves a multiple half protocols with potential for higher contrast media and radiation doses. DECT can play an important role in vascular imaging by up quality of the image with fewer contrast media and lower radiation dose compared to plain CTA (Figure 9).

Since tissues attenuate further of the X-ray beam at photons energies nearer to their K-edge, low energy DECT with custom VMC pictures can increase the attenuation of intravascular iodine whereas decreasing the contrast media bolus. in addition, radiation dose square measure usually significantly diminished by utilizing virtual unenhanced pictures, which might eliminate the need for multiphasic imaging. These blessings of DECT can enhance CTA protocols and procure exquisite image quality what is more. Such pictures would possibly even be a lot of improved by implementing calcium subtraction techniques at post-processing [28].

\section{Artifact reduction}

Artifact reduction is another potential advantage of DECT. Interpretation of typical CT may even be compromised at intervals the setting of beam hardening and photons starvation artifacts caused by metallic prostheses. Beam hardening generally happens due to attenuation of low energy photons among a polychromatic X-ray beam that contributes to scatter radiation and whole but to not a quality of the image. By method of meter half (VMC) pictures via DECT acquisition, these low energy photons square measure most well-liked to be removed, which thus, eliminates their contribution to noise and whole. a lot of whole reduction square measure typically achieved through the applying of a software that reduces metal whole. the whole reduction is clinically useful once analysis of, let's say, the pelvis is prohibited
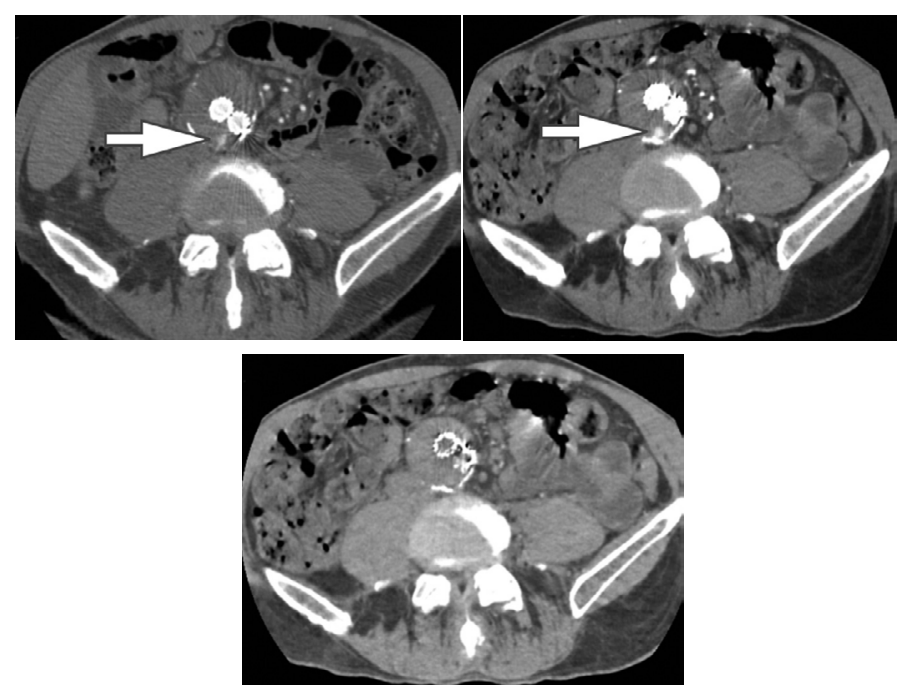

Figure 9. A 77-year-old man with endoleak following tubing graft repair of academic degree abdominal aneurysm. (A) Single energy CT at one hundred forty $\mathrm{kVp}$ shows the endoleak among the aneurysm sac (arrow). (B) Follow up twin energy CT virtual monochromatic image at sixty-five Kev with half-hour reduction of IV contrast media load demonstrates no loss of image quality and a further conspicuous look of the endoleak (arrow). in addition, note reduction among the degree of streak artifact from the tubing graft. (C) in addition to reducing contrast media load and artifact, twin energy $\mathrm{CT}$ in addition permits acquisition of virtual unenhanced pictures, negating the need for a separate true unenhanced acquisition. by beam hardening and streak whole concerning a hip restorative. it's valuable in tube imaging, where iodine pictures can facilitate reduce streak whole at intervals the assessment of patency of the vessels and endoleak detection in patient's square measure treated with coil and/ or embolization.

\section{Limitations of DECT}

DECT is clearly useful and up-coming, but it is a relatively young imaging modality and extra analysis is needed on many aspects to clarify and guarantee its clinical applications. In spite of the necessary enhancements inside the first sequential-scan approaches to DECT, low-energy data sets generally suffer from poor contrast-to-noise ratios and yield higher image noise on virtual unenhanced pictures of sex organs compared to those seen on unenhanced pictures in typical contrast-enhanced CT scan series. Also, obese patients may have higher radiation doses to realize spare image quality. Virtual unenhanced DECT pictures can delete small viscus stones in iodinesubtraction post method, and incomplete iodine subtraction can cause false-positive findings on virtual unenhanced pictures. DECT data sets are significantly larger than those sometimes seen in ancient singleenergy CT scans. However, computing capability, speed, and storage are up quickly and are unlikely to represent lasting barriers to adopt high data-demanding DECT scan acquisition and post method.

\section{Conclusion}

Dual-energy CT offers exciting Advanced and sophisticated applications that were previously inaccessible with typical single energy CT. The potential edges of DECT embrace exaggerated detection and characterization of a lesion, near-accurate medication staging and analysis of treatment response, and reduction of artifacts, all at comparable or maybe reduced radiation doses.

\section{References}

1. Brenner DJ (2010) ought to we have a tendency to worry concerning the speedy increase in CT usage? Rev ring Health 25: 63-68.

2. Henzler T, Fink C, Schoenberg SO, Schoepf UJ (2012) Dual-energy CT: radiation dose aspects. AJR Am J Roentgenol 199: S16-S25. [Crossref]

3. American state (2011) Physical background. In: Johnson TRC, Fink C, Schönberg therefore, Reiser MF, Eds. twin Energy CT in Clinical observe. Berlin, Germany: Springer 3-10.

4. Johnson TRC (2010) twin energy CT: scientific proof and clinical application.

5. dramatist JG, Fidler JL (2011) Pilot multi-reader study demonstrating potential for dose reduction in twin energy viscus CT victimisation nonlinear mixing of mixed kVimage datasets. Eur Radiol 21: 655-652.

6. Thieme SF, Högl S, Johnson TRC. respiratory organ ventilation (2011) In: Johnson TRC, Fink C, Schönberg therefore, Reiser MF, eds. twin Energy CT in Clinical observe. Berlin, Germany: Springer 91-100.

7. Kim SJ, Lim HK, Lee HY (2012) Dual-energy CT within the analysis of neura structure hemorrhage of unknown origin: differentiation between growth injury and puto re hemorrhage. Am J Neuroradiol 33: 865-872.

8. Delaware (2014) Dual-energy CT of the abdomen. Abdom Imaging 39: 108-134.

9. Mallinson PI, McLaughlin P, Nicolaou S, Munk PL, Ouellette H (2014) Peering through the glare: victimisation dual-energy CT to beat the matter of metal artefacts in bone radiology. Skel Radiol 43: 567-575.

10. McCollough CH, Schmidt B, Liu X, Yu L, Leng S (2011) Dual energy algorithms and postprocessing techniques. In: Johnson TRC, Fink C, Schönberg therefore, Reiser MF, Eds. twin Energy CT in Clinical observe. Berlin, Germany: Springer 43-54.

11. Siemens (2013) twin energy CT: SOMATOM definition. Accessed Apr fifteen.

12. metal, Paden RG, Silva AC, Humphreys mister (2014) Dual-energy vs typical CAT in crucial stone composition. Urology. 
13. Mingliang Qu, Ramirez Giraldo JC, Leng S, Williams JC, Vrtiska TJ (2011) DualEnergy Dual-Source CT with Additional Spectral Filtration Can Improve the Differentiation of Non-Uric Acid Renal Stones: An Ex Vivo Phantom Study. AJR Am J Roentgenol 196: 1279-1287. [Crossref]

14. Fung GS, Kawamoto S, Matlaga BR, Taguchi K, Zhou X, et al. (2012) Differentiation of excretory organ stones victimisation dual-energy $\mathrm{CT}$ with and while not a tin filter. AJR Am J Roentgenol 198: 1380-1386. [Crossref]

15. Rat C (2011) Liver imaging. In: Johnson TRC, Fink C, Schönberg therefore, Reiser MF, Eds. twin Energy CT in Clinical observe. Berlin, Germany: Springer 145-156.

16. Simons D, Kachelriess M, Schlemmer HP (2014) Recent developments of dual-energy CT in oncology. Eur Radiol 24: 930-939. [Crossref]

17. Agrawal MD, Pinho DF, Kulkarni NM, Hahn PF, Guimaraes AR, et al. (2014) medicine applications of dual-energy CT within the abdomen. Radiographics 34: 589-612

18. Ohana M, Jeung MY, Labani A, El Ghannudi S, Roy C (2014) Pectoral twin energy CT: acquisition protocols, current applications and future developments. Diagn Interv Imag.

19. Mi-Jin Kang, Chang Min Park, Chang-Hyun Lee, Jin Mo Goo and Hyun Ju Lee (2010) Dual-Energy CT: Clinical Applications in Various Pulmonary Diseases. Radiographics 30: 685-698.

20. Geyer LL, Scherr M, Körner M (2012) Imaging of acute embolism employing a twin energy CT system with speedy kVp switching: initial results. Eur J Radiol 81: 37113718 .
21. lutetium weight unit, Zhao Y, Zhang LJ, Schoepf UJ (2012) Dual-energy CT of the respiratory organ. AJR Am J Roentgenol199: S40-S53.

22. Krissak R, Fink C (2011) respiratory organ introduction. In: Johnson TRC, Fink C, Schönberg therefore, Reiser MF, eds. twin Energy CT in Clinical observe. Berlin, Germany: Springer 83-90.

23. Coursey CA, Nelson RC, Boll DT, Paulson EK, Ho LM, et al. (2010) Dual-energy multidetector CT: how does it work, what can it tell us, and when can we use it in abdominopelvic imaging? RadioGraphics 30:1037-1055. [Crossref]

24. Lee YH, Park KK, Song HT (2012) Metal whole thing reduction in gem spec- tral imaging dualenergy CT with and with- out metal whole thing reduction software system. Eur Radiol 22:1331-1340.

25. Del Gaizo AJ, Silva AC, Hara AK (2014) The utility of dual-energy CT in abdominal imaging. Appl Radiol 43:12-19.

26. Kaza RK, Platt JF, Cohan RH, Caoili EM, Al-Hawary millimetre, et al. (2012) Dualenergy CT with single- and dual-source scanners: current applications in evaluating the sex organ tract. Radiographics 32: 353-369.

27. Schenzle JC, Sommer WH, Neumaier K (2010) Twin energy CT of the chest: however, concerning the dose? Invest Radiol 45: 347-353.

28. Schoepf UJ, Colletti PM (2012) New Dimensions in Imaging: The Awakening of DualEnergy CT. AJR Am J Roentgenol 199: S1-S2.

Copyright: $\odot 2018$ AL Mohammad MA. This is an open-access article distributed under the terms of the Creative Commons Attribution License, which permits unrestricted use, distribution, and reproduction in any medium, provided the original author and source are credited. 